\title{
La interoperabilidad de las plataformas tecnológicas de DTI como clave para el despegue de los mismos
}

\author{
Bulchand-Gidumal, Jacques \\ Universidad de Las Palmas de Gran Canaria, España \\ Instituto Universitario de Turismo y Desarrollo Económico Sostenible (TIDES) \\ jacques.bulchand@ulpgc.es
}

Pérez-Jiménez, Rafael

Universidad de Las Palmas de Gran Canaria, España Instituto para el Desarrollo Tecnológico y la Innovación en Comunicaciones (IDETIC) rafael.perez@ulpgc.es

\section{Resumen}

En los últimos años se están desarrollando proyectos de Destinos Turísticos Inteligentes (DTI) en distintos ámbitos geográficos gracias al impulso que los mismos están recibiendo desde todos los ámbitos. Desgraciadamente, en la mayoría de los casos estos proyectos se hacen con una visión individual, y sin tener en cuenta otros proyectos similares que se puedan estar desarrollando en zonas cercanas o incluso en zonas que se solapan geográficamente y que, por lo tanto, serán proyectos que deberán integrarse en el futuro. En este artículo se presenta una propuesta metodológica de los pasos a llevar a cabo para desarrollar una adecuada interoperabilidad entre las plataformas de DTI de un territorio geográfico. También se realiza un resumen de los resultados esperados si se aplica la misma.

Palabras clave: interoperabilidad, plataformas TIC, DTI

\section{Abstract}

In recent years, projects of Smart Tourist Destinations (STD) have been developed in different geographical areas due to the great interest that these type of projects are receiving from all areas. Unfortunately, in most cases these projects are done with an individual vision, without taking into account other similar projects that may be developing in nearby areas or even in areas that overlap geographically and that will therefore be projects which need to be integrated in the future. This article 
Actas del Seminario Internacional Destinos Turísticos Inteligentes:

nuevos horizontes en la investigación y gestión del turismo

Universidad de Alicante, 26 y 27 de octubre de 2017

presents a methodological proposal of the steps to be taken to develop an adequate interoperability among the STD platforms of a geographic territory. A summary of the expected results is also made if this methodology is applied.

\section{Introducción}

El desarrollo de sistemas tecnológicos para dar soporte a los Destinos Turísticos Inteligentes (DTI) está recibiendo un gran impulso desde el ámbito público, tanto mediante financiación nivel regional, estatal, e incluso como parte de las líneas prioritarias en el programa H2020. Pero hay que tener en cuenta que los destinos turísticos que reciben esta financiación para desarrollar su plataforma de DTI no son independientes unos de otros, por un lado, y que, además, suele haber organizaciones que tienen competencias sobre la gestión de varios destinos simultáneamente o bien interés en una pequeña parte del destino.

Así, si tomamos un destino turístico compuesto por un grupo de playas, probablemente este destino, a su vez, pertenezca al conjunto de destinos de una provincia, de una comunidad autónoma y de nivel nacional. Y, por otro lado, los municipios a los cuales pertenezcan cada una de las playas estarán interesados, principalmente, en esas playas que pertenecen al municipio.

Por ejemplo, en el caso de la isla de Gran Canaria, la zona turística de referencia se extiende por la zona sur de la isla, desde Bahía Feliz hasta el Puerto de Mogán. Esta zona comprende más de 10 zonas de playa (Bahía Feliz, San Agustín, Playa del Inglés, Maspalomas, Meloneras, etc.) que pertenecen, a su vez, a dos municipios (San Bartolomé de Tirajana y Mogán). A su vez, dentro de la misma isla también es un destino turístico importante la capital (Las Palmas de Gran Canaria, en la zona norte) que pertenece a otro municipio. Por último, la isla de Gran Canaria pertenece a la Comunidad Autónoma de Canarias.

En esta situación, la interoperabilidad de estas plataformas de DTI desarrolladas por cada destino se vuelve una cuestión crucial para garantizar que estas inversiones consiguen hacer realmente inteligentes a los destinos y se maximiza su rendimiento. Esta interoperabilidad no debe garantizarse solo entre plataformas de DTI sino también con las plataformas de ciudades inteligentes que se están desarrollando.

Además, las mencionadas plataformas deben asegurar la interacción con los sistemas preexistentes y su interoperabilidad con otras plataformas, para 
Actas del Seminario Internacional Destinos Turísticos Inteligentes:

nuevos horizontes en la investigación y gestión del turismo

Universidad de Alicante, 26 y 27 de octubre de 2017

así cumplir el objetivo de permitir el acceso eficiente de las partes interesadas (los stakeholders) a los datos y a los procedimientos de control, de manera transparente y transversal. Entre los principales stakeholders citamos a los usuarios residentes locales, los turistas, las empresas y emprendedores que operan en el territorio y, por supuesto, las administraciones en sus distintos niveles.

En el caso antes mencionado de la isla de Gran Canaria, se han identificado los siguientes proyectos o propuestas de proyecto dentro del entorno de DTI o de ciudades inteligentes:

- LPA_GC: Modelo de Ciudad Inteligente, en la ciudad de Las Palmas de Gran Canaria.

- LPA Inteligencia Azul, en la ciudad de Las Palmas de Gran Canaria.

- Las Palmas de Gran Canaria, destino turístico inteligente, en la ciudad de Las Palmas de Gran Canaria.

- Proyecto Smartdest, en el que participa la isla de Gran Canaria.

- Alianza de Municipios Turísticos de Sol y Playa, en la que participa el Ayuntamiento de San Bartolomé de Tirajana.

- Proyecto Destino Turísitico Inteligente, en los municipios de San Bartolomé de Tirajana y Mogán.

La anterior relación no excluye que en futuro cercano pueda desarrollarse un proyecto a nivel regional (Islas Canarias) o específico para alguno de los destinos turísticos compuestos por un subconjunto de las playas y zonas costeras mencionadas.

Parece lógico considerar que es necesario abordar los anteriores proyectos con una perspectiva global e integradora, que permita reducir la duplicidad de esfuerzos y desarrollos, al mismo tiempo que se garantiza que los datos, procesos y resultados de las distintas plataformas comparten algunas características básicas que faciliten que los agente que necesiten acceder a datos de más de una plataforma lo puedan hacer de manera razonable y eficiente.

Es necesario recalcar que una necesidad similar a la aquí descrita se produjo hace aproximadamente una década en el ámbito de las plataformas de administración electrónica (Otjacques, Hitzelberger y Feltz, 2007). Antes de que se estandarizaran determinados servicios horizontales (por ejemplo, la firma electrónica) el ciudadano se veía obligado a disponer de una firma digital distinta para usar cada una de las plataformas existentes, siendo casi 
Actas del Seminario Internacional Destinos Turísticos Inteligentes:

nuevos horizontes en la investigación y gestión del turismo

Universidad de Alicante, 26 y 27 de octubre de 2017

imposible la interoperabilidad y el intercambio de documentos entre plataformas. A medida que se fueron teniendo en cuenta los modelos de interoperabilidad y las arquitecturas abiertas y se estandarizaron determinados servicios, esta situación mejoró significativamente.

En este artículo, presentamos una propuesta metodológica de los pasos a llevar a cabo para desarrollar una adecuada interoperabilidad entre las plataformas de destinos y ciudades inteligentes (DCl) de un territorio geográfico.

\section{Revisión de la literatura}

Panetto y Cecil (2013) definen la interoperabilidad como un modelo en el que se produce la coexistencia, autonomía y federalismo de distintos sistemas de información, que funcionan de forma autónoma y con un acoplamiento ligero entre ellos. Es decir, que pueden intercambiar información sin perder su lógica individual de funcionamiento.

En este sentido, Sheth (1999) plantea la existencia de tres niveles de interoperabilidad:

- De sistemas y datos, lo que el autor denomina de primera generación. Se trata de un modelo en el que la interoperabilidad se busca a nivel de la tecnología, las bases de datos y los protocolos tecnológicos.

- De sistemas, datos e información, o de segunda generación. En este caso, el énfasis se encuentra en la estructura de los datos y su sintaxis.

- De sistemas, datos, información, conocimiento (incluyendo la parte social) y procesos, o de tercera generación. En este caso se busca la interoperabilidad semántica, es decir, entender los conceptos a nivel de dominio y realizar la conexión en un modelo de orquestación entre distintas plataformas de alto nivel de ámbito empresarial o geográfico, teniendo en cuenta la más que probable heterogeneidad entre estas plataformas.

De cara a la propuesta que aquí se presenta, se considera fundamental aspirar a lograr el nivel máximo, pues la gestión de los DTI involucra no solo la gestión de sistemas y datos, sino también el conocimiento y procesos necesarios para la gestión del destino.

Esta última es la que muchas veces se denomina interoperabilidad semántica, que ha sido definida como aquella que permite a las entidades 
Actas del Seminario Internacional Destinos Turísticos Inteligentes:

nuevos horizontes en la investigación y gestión del turismo

Universidad de Alicante, 26 y 27 de octubre de 2017

cooperantes salvar los conflictos que puedan existir de significados distintos, perspectivas y supuestos, mediante la creación de un entorno de información compatible semánticamente basado en el acuerdo entre las entidades acerca de los significados de los conceptos (Park y Ram, 2004).

\section{Metodología propuesta}

En función de lo descrito, se propone una metodología cuyo objetivo es lograr la interoperabilidad semántica entre las plataformas de $\mathrm{DCl}$, permitiendo así el intercambio de información, conocimiento y procesos entre los destinos, al mismo tiempo que se garantiza la consolidación de la información hacia niveles superiores de cara a facilitar la gestión de los mismos por parte de las organizaciones supra-territorio. Al mismo tiempo, también es fundamental garantizar el acceso a la información por parte de todos los agentes implicados.

La metodología se sustenta en las siguientes tres fases y subfases:

1. Diagnóstico de la situación de implantación de las plataformas de DCI en el área de interés.

1.1. Categorización de servicios por tipología de territorios para los modelos de $\mathrm{DCl}$.

1.2. Valoración del estado actual de implantación de servicios e infraestructuras de $\mathrm{DCl}$ en los territorios objetivo y en espacios similares.

1.3. Priorización de servicios para su interoperabilidad.

2. Propuesta de estrategias de interacción entre las plataformas de DCl.

2.1. Definición de estrategias de interoperabilidad a emplear.

2.2. Modelos de coexistencia con soluciones ya implantadas.

2.3. Definición de formatos de interacción entre plataformas.

3. Definición de formatos de presentación de información a los stakeholders del proyecto.

3.1. Definición del tipo de información y el formato de los interfaces para cada tipo de usuario de los sistemas de DCl.

3.2. Validación con los distintos tipos de actores implicados.

3.3. Puesta en marcha de los servicios seleccionados.

A continuación se describen estas fases y subfases en detalle. 
Actas del Seminario Internacional Destinos Turísticos Inteligentes:

nuevos horizontes en la investigación y gestión del turismo

Universidad de Alicante, 26 y 27 de octubre de 2017

3.1. Fase 1. Diagnóstico de la situación de implantación de las plataformas de $D C l$ en el área de interés

Esta fase tiene como objetivo obtener una evaluación del estado actual de la cuestión desde diversas facetas, definiendo las soluciones presentes o en proceso de desarrollo tanto en los territorios-objetivo como en otros con características similares.

Para ello plantea definir el escenario inicial en el que debe desarrollarse el proyecto de integración. Debe incluir no sólo las iniciativas propuestas dentro de los grandes proyectos financiados por los gobiernos sino también aquellas susceptibles de incorporarse a estos modelos. Además, se estudiarán las soluciones utilizadas en otras regiones.

Finalmente, dado que territorios de categorías similares con problemáticas parecidas, pueden agruparse definiendo y acotando sus puntos de encuentro, se hará este proceso de agrupación para de esta forma determinar soluciones semejantes que ayude a las administraciones implicadas a optimizar sus recursos y facilitar su gestión.

\subsection{Categorización de servicios por tipología de territorios para los modelos de $\mathrm{DCl}$}

Los territorios-objetivo, si bien presentarán aspectos comunes, también tendrán una amplia diversidad de escenarios, tanto rurales como urbanos, a partir de referencias relacionadas con las formas de regulación y planificación tanto a nivel territorial, como urbanística, la densidad y distribución de población, tamaño de la isla, presencia de espacios naturales protegidos, actividad económica, etc.

Por ello, se considera adecuado realizar una primera taxonomía que categorice los territorios a partir de sus tipologías, para buscar espacios con suficientes elementos de coincidencia como para definirlos como relativamente homogéneos, logrando así reconocer de manera común tanto los problemas, como las propuestas de mejora y soluciones que hacen posibles el intercambio de unas y otras como consecuencia de las similitudes determinadas y definidas. 
Actas del Seminario Internacional Destinos Turísticos Inteligentes:

nuevos horizontes en la investigación y gestión del turismo

Universidad de Alicante, 26 y 27 de octubre de 2017

1.2. Valoración del estado actual de implantación de servicios e infraestructuras de $\mathrm{DCl}$ en los territorios objetivo y en espacios similares

Actualmente se han producido múltiples iniciativas para crear espacios inteligentes conectados, tanto en zonas urbanas como a nivel de zonas geográficas delimitadas. Además de las convocatorias públicas de ciudades y destinos inteligentes, hay iniciativas a nivel de gobierno regional, provincial, insular, comarcal y municipal que pretenden dotar las infraestructuras básicas, o que han desarrollado aplicaciones que pueden incorporarse posteriormente a una malla de sociedad digital. Esta tarea pretende trazar un mapa de situación de la implantación en los territorios-objetivo de este tipo de soluciones.

También se deberán encontrar soluciones y propuestas que se están barajando para otros territorios que compartan todas o algunas de las características analizadas en el punto anterior.

\subsection{Priorización de servicios para su interoperabilidad.}

Tras un proceso de aproximaciones sucesivas, filtrado y posterior depuración, se establecerán los niveles de prioridad de para cada uno de los servicios encontrados. Esto se basará en el establecimiento de una métrica que permitirá clasificar en función de su utilidad (percibida por los usuarios), su impacto (económico, medioambiental, territorial, social etc.) y su coste (en términos de retorno de la inversión y la disponibilidad o no de la infraestructura necesaria para su implantación). Todos los agentes implicados podrán entonces utilizar estas propuestas para integrar de forma coherente y homogénea las problemáticas y los servicios comunes para cada uno los territorios. Basándose en dicha métrica se establecerá el conjunto de indicadores que permitan medir la eficiencia de uso de los servicios priorizados.

\subsection{Fase 2. Propuesta de estrategias de interacción entre las plataformas de $\mathrm{DCl}$}

Esta fase define las estrategias a seguir para asegurar el acceso a la información entre las distintas plataformas que puedan implementarse (a nivel regional, provincial, insular, comarcal y municipal), de modo que los distintos colectivos (residentes permanentes, turistas y residentes estacionales, empresas y administraciones) puedan conocer los datos que ofrezcan las citadas plataformas de un modo ubicuo. 
Actas del Seminario Internacional Destinos Turísticos Inteligentes:

nuevos horizontes en la investigación y gestión del turismo

Universidad de Alicante, 26 y 27 de octubre de 2017

Para ello se parte de la selección de la estrategia de interoperabilidad a actuar, en función de las propuestas que se estén implantando y de la máxima sencillez en la solución adoptada, tomando como idea base la estructura de gestor de canales (channel manager), habitualmente utilizada en el sector turístico.

De esta manera se permitirá:

- Publicar de una manera compacta y accesible los datos de cada una de las administraciones implicadas, mejorando de esta manera el acceso a los indicadores para los agentes implicados.

- Mejorar la eficiencia y la transparencia.

- Aumentar la dimensión transversal (tanto horizontal y vertical) de las inversiones que se realicen, asegurando su integración en una estructura de información accesible.

- Mejorar la gobernanza de las zonas objetivo al aumentar tanto el impacto de las inversiones y su visibilidad por parte de los administrados.

La interconexión de estructuras para el intercambio de información seguirá una arquitectura de integración jerárquica de abajo a arriba, partiendo de las soluciones existentes (donde las haya) o proponiendo estructuras sostenibles en aquellos lugares que aún no las hayan implementado.

\subsection{Definición de estrategias de interoperabilidad a emplear}

El primer paso consiste en la definición del modelo de interoperabilidad a emplear. En general, en el ámbito de la interoperabilidad, y en un proyecto como el de este tipo, se conciben dos posibles modelos:

- Adaptar los sistemas ya existentes e incluir como parte de los que se van a desarrollar en el futuro el hecho de que ofrezcan la información en un formato estándar de cara a que se puedan integrar, comparar y utilizar estos datos por otras plataformas.

- Hacer que el sistema que va a permitir el proceso de interoperabilidad se encargue de ir a buscar los datos a cada uno de los sistemas existentes, sea cual sea su estructura y formato, llevándolos hacia una base de datos común. 
Actas del Seminario Internacional Destinos Turísticos Inteligentes:

nuevos horizontes en la investigación y gestión del turismo

Universidad de Alicante, 26 y 27 de octubre de 2017

En esta metodología se sugiere como preferible la segunda de las estrategias, ya que se entiende que será tremendamente complicado forzar a que cada uno de los sistemas existentes (y los que se vayan a desarrollar en el futuro) acepten y adopten un determinado estándar. El caso que se plantea aquí es similar al existente actualmente en el sector alojativo turístico, en el que los alojamientos cuentan con sistemas de gestión de la propiedad (PMS) creados por fabricantes distintos, usando diversas bases de datos y estructuras de datos no directamente compatibles. En el proceso de volcar la disponibilidad en los respectivos canales de venta (CRS), han aparecido los denominados channel managers o gestores de canales, que son capaces de leer la información de los distintos PMS y volcar la misma en distintos CRS, permitiendo que sistemas muy diversos entre sí puedan comunicarse en tiempo real. En esta tarea se revisará la aplicabilidad general de esta hipótesis para comprobar si es necesario establecer estrategias alternativas ad-hoc para ciertos servicios.

\subsection{Modelos de coexistencia con soluciones ya implantadas}

En muchos casos, los servicios a generar dentro de una plataforma de ciudad/destino inteligente son proporcionados por entes integrados en el territorio (ayuntamientos, empresas públicas y/o privadas, asociaciones, centros formativos o de investigación...), que en algunos casos cuentan con servicios preexistentes que es necesario integrar en las plataformas, o bien tienen carencias en cuanto a infraestructura de comunicaciones, o requieren un esfuerzo a los agentes implicados que hará difícil en el futuro mantener las estructuras y los datos actualizados en el futuro, comprometiendo la sostenibilidad de la propuesta.

Es por ello que se propone en este momento revisar la tabla de servicios y necesidades prioritarias según tipologías de territorio definidos en la Fase 1. Así, se estudiarán los requisitos que se deben cumplir para asegurar la interconexión dentro de un modelo de territorio digital accesible y sostenible en el tiempo, tanto en el caso de servicios preexistentes, como en el caso de servicios para los que ya está prevista la implantación de plataformas en breve, o de servicios que se desee planificar para su puesta en marcha en el futuro. 
Actas del Seminario Internacional Destinos Turísticos Inteligentes:

nuevos horizontes en la investigación y gestión del turismo

Universidad de Alicante, 26 y 27 de octubre de 2017

2.3. Definición de formatos de interacción entre plataformas

Se definen los modelos concretos de interoperabilidad de acuerdo con las plataformas, tanto si ya está prevista la implantación de plataformas de territorio inteligente, como si se desea planificarla para su desarrollo en el futuro. Para ello se parte de que la capacidad de acción sobre los modelos comerciales es limitada, pero se entiende que se puede obtener acceso a los datos que proporcionan y agruparlos según un formato común.

Una vez establecidos los criterios de compartición de información entre plataformas, el último paso es asegurar la compatibilidad de la información obtenida con las redes de comunicación convencionales con el fin de permitir el acceso ubicuo.

\subsection{Fase 3. Definición de formatos de presentación de información a los stakeholders del proyecto}

Una de las principales características de los proyectos de ciudades y destinos inteligentes es la gran cantidad de datos e información que forman parte de los mismos. Los datos provienen tanto de fuentes internas del propio territorio (sensores de tráfico y medioambientales, sistemas de depuración de aguas, recogida de basuras, etc.) como de fuentes externas (datos macroeconómicos, datos microeconómicos, entradas de turistas, índices de ocupación hotelera, etc.). Normalmente, estos datos, a su vez, se procesan de cara a obtener distintos tipos de información que posteriormente será utilizada en la toma de decisiones.

Adicionalmente, es previsible que esta cantidad de datos se vea incluso incrementada al dotar a las regiones de herramientas de interoperabilidad, ya que ello permitirá, potencialmente, que se produzca un intercambio de información entre dichos territorios pudiendo realizar comparativas, índices, etc.

Por último, destacar que habitualmente el tipo de información que utilizará cada uno de los agentes indicados será distinta. Por ejemplo, el turista o residente temporal puede tener interés en datos relativos a temas patrimoniales y a la calidad del aire. El residente permanente, a cuestiones relativas al tráfico y al reciclaje de basuras. Las administraciones, a todo tipo de datos como aquellos relativos a tráfico, calidad del aire, monitorización de variables que indican posibles riesgos, etc. $Y$ las empresas que operan en el 
Actas del Seminario Internacional Destinos Turísticos Inteligentes:

nuevos horizontes en la investigación y gestión del turismo

Universidad de Alicante, 26 y 27 de octubre de 2017

territorio, a datos que les permitan ofrecer, a su vez, productos y servicios para las personas que pasan por dicha zona.

Destacar que los resultados de esta fase deben servir incluso para aquellos territorios en los que aún no haya definido proyectos de territorio inteligente, de forma que puedan explotar y presentar la información que ya tengan disponible, así como tener acceso a comparativas de datos de los territorios que sí tienen proyectos de este tipo.

3.1. Definición del tipo de información y el formato de los interfaces para cada tipo de usuario de los sistemas de DCl

La gran cantidad de datos e información que se obtiene de las plataformas de territorios inteligentes debe hacerse accesible para que pueda ser utilizada por los agentes involucrados en la toma de decisiones en los distintos niveles (el usuario local, el turista, el residente temporal, las empresas que operan en el territorio y las administraciones), sus formatos e interfaces de presentación también deben ser los adecuados para cada uno de ellos. Por ello, el principal objetivo de esta fase será analizar y definir estos tipos y formatos de información.

\subsection{Validación con los distintos tipos de actores implicados}

Se trata de validar los resultados del punto 3.1 con los perfiles destinatarios de los mismos. Este proceso, de definición del formato de datos, presentación al usuario para su validación y refinamiento del mismo en función de lo aprendido se llevará a cabo varias veces para intentar disponer de una serie de interfaces lo más cercano posible a las necesidades finales de los agentes involucrados. La metodología a emplear en esta fase será iterativa: entrevistas en profundidad con los agentes implicados, definición de formatos, validación de los mismos con los agentes y aprendizaje, comenzando de nuevo el proceso.

\subsection{Puesta en marcha de los servicios seleccionados}

Tras la categorización realizada en la Fase 1, se seleccionará un grupo de servicios representativos de todos los catalogados. Deberán cumplir las características de ser de interés para todos los tipos de territorios definidos y de ser servicios en los que el proceso de interoperabilidad resulte especialmente valioso e interesante. Para estos servicios, se procederá a analizar: 
Actas del Seminario Internacional Destinos Turísticos Inteligentes:

nuevos horizontes en la investigación y gestión del turismo

Universidad de Alicante, 26 y 27 de octubre de 2017

- Su estado de implementación o, en su caso, su proyección de implementación, en cada una de las plataformas en curso de desarrollo y su estado en el caso de los territorios que no cuenten con desarrollo en este ámbito.

- Su lenguaje de definición, sus procesos asociados y su estructura de datos de cara a estudiar las necesidades de interoperabilidad en cada uno de los niveles.

- Definición de cómo se va a llevar a cabo la interoperabilidad entre todos los agentes generadores de los datos.

- Definición de los formatos de presentación de los datos.

\section{Resultados esperados}

El uso de una metodología como la propuesta permitirá que se obtengan una serie de beneficios para todos los agentes participantes. Entre todos los esperados, citamos los siguientes:

- Una mejora en la eficacia de la inversión por parte de las administraciones, al proporcionar una base de estudio sobre los servicios prioritarios a integrar en iniciativas de digitalización de servicios a nivel local o insular.

- La posibilidad de publicar de una manera compacta y accesible los datos de cada una de las administraciones implicadas.

- Una mejora de la eficiencia y la transparencia de las administraciones al hacer la información accesible de forma ubicua.

- Un aumento de la dimensión transversal (tanto horizontal y vertical) de las inversiones que se realicen, asegurando su integración en una estructura de información accesible.

- Una mejora de la gobernanza de los territorios objetivo.

Además de todo lo anterior, la puesta en marcha de una metodología orientada a integrar e interoperar las distintas plataformas que se vayan desarrollando en el ámbito de los DCI permitirá, principalmente, lograr que realmente los territorios sean inteligentes. No se puede concebir que un territorio pueda definirse como inteligente de forma aislada e independiente de su entorno y de otros territorios que lo rodean. 
Actas del Seminario Internacional Destinos Turísticos Inteligentes:

nuevos horizontes en la investigación y gestión del turismo

Universidad de Alicante, 26 y 27 de octubre de 2017

\section{Discusión}

El presente artículo pretende introducir en el proceso de construcción de los DTI un nuevo elemento de reflexión y debate: el relativo a la necesidad de garantizar la interoperabilidad de las plataformas. En este sentido, se apunta una metodología que pretende servir para garantizar la interoperabilidad en ámbitos territoriales en los cuales puede haber, simultáneamente, destinos que ya dispongan de plataformas tecnológicas para la construcción de sus DTI junto a otros que estén aun en fases de conceptualización y otros que ni siquiera se estén planteando su desarrollo. Adicionalmente, también se tendrán en cuenta los proyectos de ciudad inteligente que se estén llevando a cabo en la zona geográfica que se considere.

El objetivo final debe ser garantizar y mejorar la gobernabilidad de los destinos, facilitando a todos los agentes implicados (administraciones, empresas, emprendedores potenciales, residentes y turistas) tanto la mejora de la gestión como el simple acceso al conocimiento existente.

Dicho de otra forma, lograr no un DTI sino un ecosistema turístico inteligente (Gretzel et al., 2015), definido como un sistema turístico que utilice las tecnologías inteligentes para crear, gestionar y proporcionar experiencias y servicios turísticos caracterizados por una compartición intensiva de información y de co-creación de valor.

\section{Referencias}

Gretzel, U., Werthner, H., Koo, C., \& Lamsfus, C. (2015). Conceptual foundations for understanding smart tourism ecosystems. Computers in Human Behavior, 50, 558-563.

Otjacques, B., Hitzelberger, P., \& Feltz, F. (2007). Interoperability of e-government information systems: Issues of identification and data sharing. Journal of Management Information Systems, 23(4), 29-51.

Panetto, H., \& Cecil, J. (2013). Information systems for enterprise integration, interoperability and networking: theory and applications. Enterprise Information Systems - Information Systems for Enterprise Integration, Interoperability and Networking: Theory and Applications. Volume 7 Issue 1, February 2013, pages 1-6

Park, J., \& Ram, S. (2004). Information systems interoperability: What lies beneath?. ACM Transactions on Information Systems (TOIS), 22(4), 595-632. 
Actas del Seminario Internacional Destinos Turísticos Inteligentes: nuevos horizontes en la investigación y gestión del turismo Universidad de Alicante, 26 y 27 de octubre de 2017

Sheth, A. P. (1999). Changing focus on interoperability in information systems: from system, syntax, structure to semantics. In Interoperating geographic information systems (pp. 5-29). Springer US. 